\title{
A certified reference material for the scratch test
}

\author{
R. Jacobs ${ }^{\mathrm{a}, *}$, J. Meneve ${ }^{\mathrm{a}}$, G. Dyson ${ }^{\mathrm{b}}$, D.G. Teer ${ }^{\mathrm{b}}$, N.M. Jennett ${ }^{\mathrm{c}}$, P. Harris ${ }^{\mathrm{c}}$, J. von Stebut ${ }^{\mathrm{d}}$, \\ C. Comte ${ }^{\mathrm{d}}$, P. Feuchter ${ }^{\mathrm{e}}$, A. Cavaleiro ${ }^{\mathrm{f}}$, H. Ronkainen ${ }^{\mathrm{g}}$, K. Holmberg ${ }^{\mathrm{g}}$, U. Beck ${ }^{\mathrm{h}}$, G. Reiners ${ }^{\mathrm{h}}$, \\ C.D. Ingelbrecht ${ }^{\mathrm{i}}$ \\ ${ }^{a}$ Materials Technology Centre, Flemish Institute for Technological Research - VITO, Boeretang 200, B-2400 Mol, Belgium \\ ${ }^{\mathrm{b}}$ Teer Coatings Ltd - TCL, 290 Hartlebury Trading Estate, Hartlebury, Kidderminster, Worcestershire DY10 4JB, UK \\ ${ }^{\mathrm{c}}$ National Physical Laboratory - NPL, Materials Centre, Queens Road, Teddington, Middlesex TW11 OLW, UK \\ ${ }^{\mathrm{d}}$ Laboratoire de Science et Génie des Surfaces - LSGS, Ecole des Mines, Parc de Saurupt, F-54042 Nancy Cedex, France \\ ${ }^{\mathrm{e}}$ Mössner Gmbh - Hamödia, Diamantwerkzeugfabrik, Kelterstrasse 82, D-75179 Pforzheim, Germany \\ fInstituto Pedro Nunes - IPN, Univ. Coimbra, Polo II, Pinhal de Marrocos, 3030 Coimbra, Portugal \\ ${ }^{\mathrm{g}}$ VTT Industrial Systems - VTT, Metallimiehenkuja 6, Espoo, P.O. Box 1702, FIN-02044 VTT, Finland \\ ${ }^{\mathrm{h}}$ Bundesanstalt für Materialforschung - BAM, Div. VIII.2 Surface Technologies, Unter den Eichen 87, D-12200 Berlin, Germany \\ iEuropean Commission, Joint Research Centre, Institute for Reference Materials and Measurements, Retieseweg, B-2440 Geel, Belgium
}

\begin{abstract}
Adhesion and other mechanical behaviour of coatings is at present routinely tested in industry and research organisations using the scratch test. The test has been the subject of a European Standard prEN 1071-3:2000 established by the European Standards Committee CEN TC184 WG5. A previous European project on the development and validation of test methods for thin hard coatings-FASTE-revealed that uncertainties in the Rockwell C stylus tip shape represent a major source of error for the scratchtest method. Therefore a follow-up project-REMAST-was started to develop and certify a reference material as a quality control tool for the testing and qualification of scratch-test instruments. In addition, a considerable effort was devoted to improve the scratch stylus manufacturing process. This paper presents the results of the REMAST project. During a feasibility study, two candidate materials - titanium nitride (TiN) and diamond-like carbon (DLC) coatings both on high speed steel substrates-were evaluated. Because of the lower sensitivity to styli tip shape variations and higher data scatter observed for the TiN coating, DLC was chosen as the reference material to be certified. One thousand samples were produced, as well as qualified styli to carry out the certification campaign, including homogeneity and stability testing. Nine independent laboratories were involved in the certification exercise, and the data were statistically analysed to obtain the certified critical load values and their uncertainty ranges. A certified reference material BCR-692 is available for verification purposes. This presents three repeatable failure events at certified critical load intervals and can provide a good indication of overall scratch-test instrument performance, including stylus condition and calibration. It is also useful as a diagnostic tool, providing a means of sensitive monitoring of machine and stylus performance over extended periods. Considerable improvement of the quality of scratch styli was achieved by the strict control of all manufacturing steps.
\end{abstract}

(C) 2003 Elsevier Science B.V. All rights reserved.

Keywords: Adhesion; Scratch test; Certified reference material

\section{Introduction}

Coating adhesion can be tested using the quick and simple 'scratch test' in which a diamond stylus is drawn across a surface with increasing normal load until well defined failures occur, at critical loads $\mathrm{Lc}_{1}, \mathrm{Lc}_{2}, \ldots, \mathrm{Lc}_{n}$. The failure events are detected by examination of the

\footnotetext{
*Corresponding author. Tel.: +32-14-335623; fax: +32-14321186.

E-mail address: ria.jacobs@vito.be (R. Jacobs).
}

scratch track after scratching using an optical microscope. The scratch stylus is usually a Rockwell C diamond with $200 \pm 10 \mu \mathrm{m}$ tip radius, and the scratchtest method is described in the (prospective) European Standard prEN 1071-3 [1].

The reliability of the scratch-test method was investigated as part of a previous European project (FASTE) [2]. The main conclusion of that work was that variation in stylus tip shape, either due to damage or incorrect radius, was the main source of uncertainty in the test 


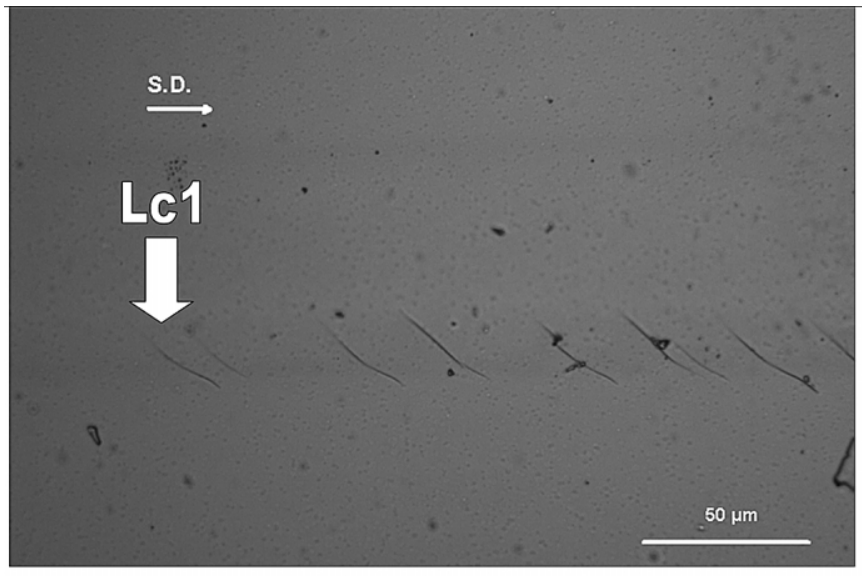

(a)

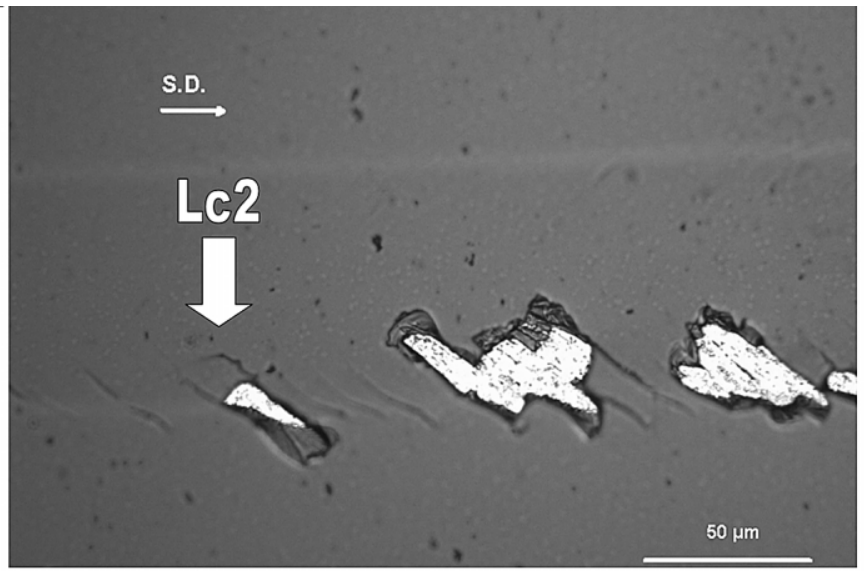

(b)

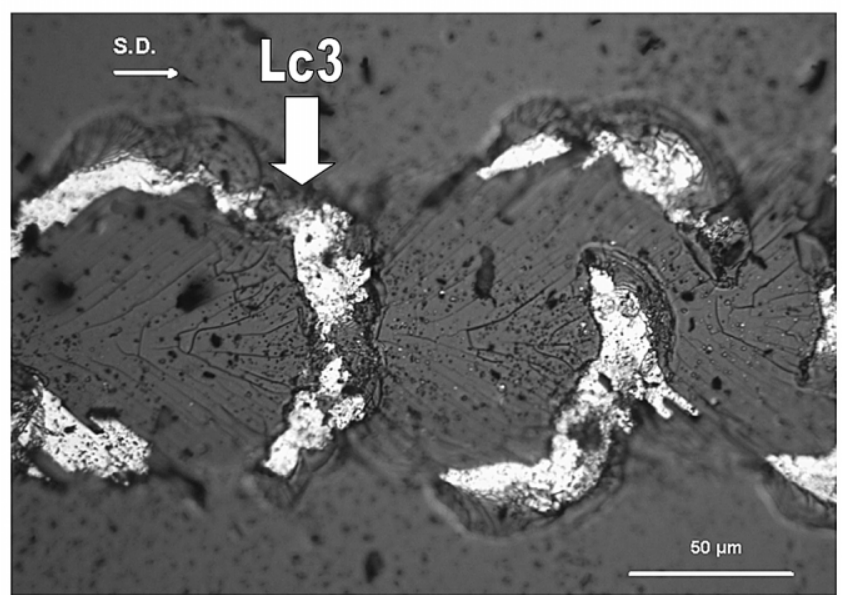

(c)

Fig. 1. Failure events associated with (a) $\mathrm{Lc}_{1}$; (b) $\mathrm{Lc}_{2}$ and (c) $\mathrm{Lc}_{3}$ (S.D., scratch direction).

method. The REMAST project described here [3] was therefore set up with the objective of developing and certifying a 'real world' reference material for the verification of the proper functioning of scratch-test instruments by detecting deviations in stylus tip shape and errors in load or displacement calibrations or other instrument malfunctions. Secondary objectives were improvements in stylus manufacturing and characterisation methods, because FASTE had shown that many styli in use deviated significantly from those specified [4] for the scratch testing method.

\section{Feasibility study and selection of candidate material}

Two candidate coating types, diamond-like carbon (DLC) and TiN, both on high speed steel substrates, were selected for the feasibility study. TiN is a very widely used and established coating type while DLC coatings represent an important emerging technology.
Scratch test styli were ground according to ISO specifications [4].

Three scratch test failure events were identified for DLC (Fig. 1): forward chevron cracks at the borders of the scratch track $\left(\mathrm{Lc}_{1}\right)$, forward chevron cracks at the borders of the scratch track accompanied by interfacial spallation $\left(\mathrm{Lc}_{2}\right)$ and gross interfacial shell-shaped spallation $\left(\mathrm{Lc}_{3}\right)$. Four failure events were identified for TiN coatings: longitudinal cracks at the track edges $\left(\mathrm{Lc}_{1}\right)$, semicircular coating cracks inside the scratch tracks $\left(\mathrm{Lc}_{2}\right)$, cohesive chipping at the tracks edges $\left(\mathrm{Lc}_{3}\right)$ and spallation at the track edges $\left(\mathrm{Lc}_{4}\right)$.

Homogeneity and stability testing, completed with data on stylus wear, allowed a comparison of the two candidate coatings.

Extensive homogeneity testing was performed to investigate potential differences within samples and between samples originating from different locations in the reactor or different coating deposition batches. The DLC samples showed lower scatter in critical loads 
compared to the TiN samples, indicating better homogeneity or more easily detected failure events. Chemical heterogeneity was studied by XPS, Auger and GD-OES. No significant heterogeneity was detected, neither for DLC nor TiN.

A series of accelerated degradation tests was carried out to determine stability during cycled exposure to high and low temperature. In addition, medium-term stability testing after storage in laboratory air for 6 months was carried out. No evidence of instability was detected for both coating types.

Wear experiments revealed that the stylus suffered from significant wear when scratching the TiN coated sample after only 50 scratches, while the expected lifetime of the stylus against DLC is over 400 scratches.

The most important factor taken into account was the sensitivity of critical load to tip radius $R$ relative to the standard deviation of the data $(\Delta \mathrm{Lc} /(\Delta \mathrm{Rs}))$. The DLC was much superior in this respect, for all critical loads.

However, the load range for DLC (up to approximately $35 \mathrm{~N}$ ) was half that of the TiN (up to $65 \mathrm{~N}$ ) resulting in a smaller probe depth $(4-6 \mu \mathrm{m})$, but this was considered sufficient for the evaluation of stylus shape, especially bearing in mind that 'multi-mode' scratch testing, now being developed [5] and likely to become the standard method in scratch testing, will use lower loads than those generally used in current practice.

On the basis of the above considerations DLC was selected as the coating to be used for the reference material.

\section{Reference material and stylus production}

\subsection{Production of the reference material}

Eleven batches of PACVD DLC coated steel coupons $30 \times 30 \times 5 \mathrm{~mm}^{3}$ of Böhler S790 powder metallurgy steel were prepared using the same method as in the feasibility study. Fig. 2 shows the reference material. Specimens were randomly selected for further testing and certification purposes.

\subsection{Production of qualified styli}

It was essential for the certification exercise to use styli conforming to the specifications for Rockwell $\mathrm{C}$ indenters [4] in order to reduce as far as possible variability in measured critical load values due to stylus irregularities or damage. Therefore, improved positioning techniques were used during stylus manufacturing, combined with the selection of appropriate wheels for preliminary grinding and polishing steps. Moreover, crystal orientations were checked by Laue diffraction before grinding. Tip radii and geometry were determined by an optical contour technique, knife-edge profilometry, interferometry and Twymann-Green interferometry. It

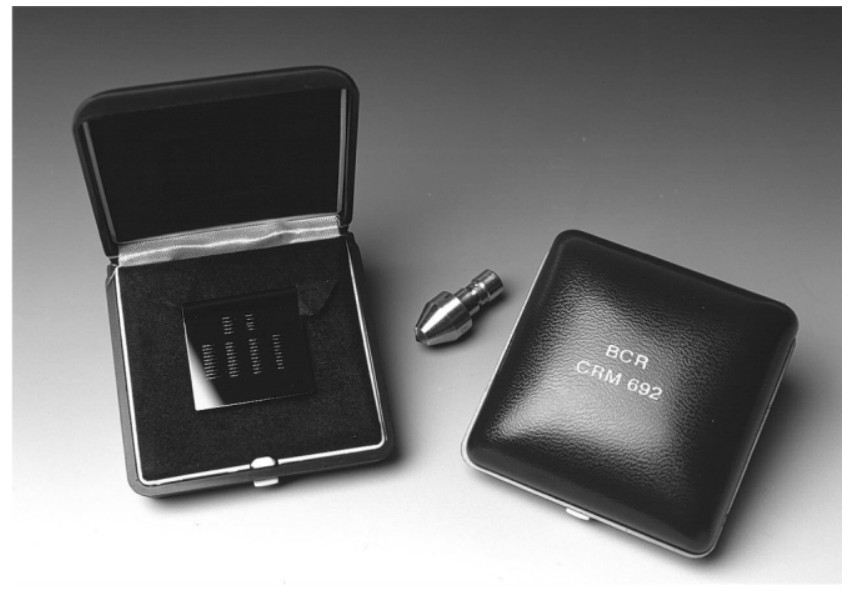

Fig. 2. The reference material BCR-692.

was found that the stylus tips often showed 'nodes' or local irregularities, with the consequence that stylus radius values depended on the tip area examined. Styli were allocated to individual laboratories for further testing and certification purposes.

\section{Certification of the reference material}

\subsection{Certified values and uncertainties}

The reference material was defined by a certified critical load value $(\mathrm{CV})$ and uncertainty $\left(U_{\mathrm{c}}\right)$ for each scratch test failure. The certified value for each critical load is the mean of means of the sets of data obtained in the certification exercise, giving equal weight to each laboratory. The expanded uncertainty is calculated according to the method given in ISO 35 [6]:

$U_{\mathrm{c}}= \pm k\left(s_{\mathrm{c}}^{2} / p+u_{\mathrm{bb}}^{2}+u_{\mathrm{bs}}^{2}+u_{\mathrm{ws}}^{2}\right)^{1 / 2}$

where $s_{\mathrm{c}}$ is the standard deviation of the set means of the certification data, $p$ is number of accepted sets, $u_{\mathrm{bb}}$, $u_{\mathrm{bs}}$ and $u_{\mathrm{ws}}$ are uncertainty components (as standard errors) related with heterogeneity (between-batches $u_{\mathrm{bb}}$, between-samples $u_{\mathrm{bs}}$ and within-samples $u_{\mathrm{ws}}$ ) and $k$ is a coverage factor.

The uncertainty calculated above represents the variation in the critical load expected from material heterogeneity and the average measurement uncertainty of the certification instruments. However, the exact critical load that an individual laboratory will obtain will be systematically offset from the certified mean value due to the specific instrument and stylus being used. The distribution of offsets arising from stylus effects $\left(u_{\mathrm{ss}}\right)$ was estimated from a stylus sensitivity study. Linear regression techniques were used to determine the distribution of offsets arising from the use of different types of machines in the certification campaign. Note that all 
Table 1

Certified values $(\mathrm{CV})$, uncertainties $\left(U_{\mathrm{c}}\right)$, and uncertainty components (N)

\begin{tabular}{llllllllll}
\hline & $u_{\mathrm{bb}}$ & $u_{\mathrm{bs}}$ & $u_{\mathrm{ws}}$ & $s_{\mathrm{c}}$ & $p$ & $u_{\mathrm{ss}}$ & $u_{1}$ & $\mathrm{CV}$ & $U_{\mathrm{c}}$ \\
\hline $\mathrm{Lc}_{1}$ & 0.40 & 0.49 & 0.55 & 1.72 & 18 & 1.05 & 0.8 & 13.6 & \pm 1.9 \\
$\mathrm{Lc}_{2}$ & 0.44 & 0.49 & 0.53 & 2.31 & 18 & 2.26 & 1.11 & 17.0 & \pm 2.0 \\
$\mathrm{Lc}_{3}$ & 0.8 & 0.81 & 1.30 & 1.78 & 14 & 1.34 & 2.11 & 28 & \pm 4
\end{tabular}

The terms $u_{\mathrm{ss}}$ and $u_{1}$ have not been included in the calculation of $U_{\mathrm{c}}$. A coverage factor $k=2$ has been applied.

of the certification instruments met the requirements of prEN 1071-3 in terms of operation and calibration, but produced different critical load values, which are assumed to be as a result of differences in mechanism or compliance. This component $\left(u_{1}\right)$ was estimated from the complete set of certification and control data made by a control lab with the same stylus as the participating lab.

The decision to exclude the stylus $\left(u_{\mathrm{ss}}\right)$ and machine $\left(u_{1}\right)$ effects from the certified range is based on the fact that these are test-related rather than material-related factors, rather less well defined than the material properties, and may well change in future due to improvements in machine performance and stylus production or to redefinition of the range of acceptable styli. The certified range, based on the materials' properties only, will not change. The terms $u_{\mathrm{ss}}$ and $u_{1}$ therefore do not appear in Eq. (1) above.

\subsection{Homogeneity of the reference material}

An extensive homogeneity study was carried out in which 6 samples from each of the deposition batches were tested ( 5 scratches per sample). A parallel study was carried out testing 3 samples from each batch (10 scratches per sample) for a more detailed examination of the 'within-sample' effect. Two-way ANOVA was then carried out for each data set (33 samples and 66 samples) to estimate the three uncertainty components (as standard uncertainties): between-batches $u_{\mathrm{bb}}$, between-samples $u_{\mathrm{bs}}$ and within-samples $u_{\mathrm{ws}}$ for each failure event (Table 1). The real within-sample effect, cannot of course be separated from the natural scatter of the method (repeatability), which also makes a contribution to $u_{\mathrm{ws}}$.

\subsection{Certification data and control data}

The nine laboratories participating in the certification campaign used three different types of instrument (CSM Revetest, VTT Industrial Systems, Teer Coatings Ltd). The instrument calibration procedures, consistent with the requirements of prEN 1071-3, included verification of sample planarity, load and load rate, horizontal displacement and displacement rate. Each laboratory was supplied with 2 samples selected randomly from the entire production and one of the qualified styli. After calibration, each laboratory made 40 scratches on each of the 2 samples with a load rate of $100 \mathrm{~N} / \mathrm{min}$, starting load $5 \mathrm{~N}$, maximum load $45 \mathrm{~N}$ and displacement rate of $10 \mathrm{~mm} / \mathrm{min}$. Critical loads $\mathrm{Lc}_{1}, \mathrm{Lc}_{2}, \mathrm{Lc}_{3}$ corresponding to the well-defined failure events were corrected for each of the calibration factors, and are used for calculation of the certified values, as given in Table 1.

In addition, the control laboratory carried out a further 5 scratches on each of the samples supplied to the participants using, in each case, the same stylus used by the respective participant and another 5 scratches using a single 'control stylus'. For each critical load, the complete set of certification data and control data $L_{\mathrm{ijk}}$ is modeled in terms of a reference value $\mu$, laboratory/ machine, stylus and sample offsets $\left(L_{\mathrm{i}}, R_{\mathrm{j}}\right.$ and $S_{\mathrm{k}}$, respectively) and random measurement error $e_{\mathrm{ijk}}$ :

$\mathrm{Lc}_{\mathrm{ijk}}=\mu+L_{\mathrm{i}}+R_{\mathrm{j}}+S_{\mathrm{k}}+e_{\mathrm{ijk}}$.

under the assumptions that the sums of the off-sets $E\left(L_{\mathrm{i}}\right)=E\left(R_{\mathrm{j}}\right)=E\left(S_{\mathrm{k}}\right)=E\left(e_{\mathrm{ijk}}\right)=0, \quad \operatorname{var}\left(e_{\mathrm{ijk}}\right)=\sigma^{2}$, with $\sigma^{2}$ an (unknown) constant, and $e_{\mathrm{ijk}}$ independent. A regression analysis was carried out and the distribution of offsets from the regression fitting were evaluated. The standard deviation of the laboratory offsets distribution is an estimate of $u_{1}$ (Table 1). Note that this uncertainty component includes contributions both from the variations in the machines and variations in lab performance and these two cannot be separated.

\subsection{Stylus sensitivity data}

A specific stylus sensitivity test was also carried out by the control laboratory, taking eight styli with radii in the range $(200 \pm 10) \mu \mathrm{m}$, as required by ISO $6508-2$ [4] and making 9 scratches with each one on a single sample. The only sources of variation in the results of this study are (a) the different styli and (b) scatter related to the precision of the testing or to small differences in the coating across the single specimen used. These two components can be separated by a oneway ANOVA, allowing the distribution of critical load values arising from stylus effects $u_{\mathrm{ss}}$ to be estimated. It was found that $\mathrm{Lc}_{2}$ appears to be more sensitive to stylus radius (higher $u_{\mathrm{ss}}$ ) than the other critical loads.

Table 1 shows that the largest component of uncertainty for $\mathrm{Lc}_{1}$ and $\mathrm{Lc}_{2}$ is the stylus-related term $u_{\mathrm{ss}}$, which confirms the high sensitivity of the test to stylus effects as observed in the FASTE program [2]. It should be remembered, of course, that the DLC coating was chosen specifically for its high sensitivity to stylus radius. Instrument/laboratory effects (the $u_{1}$ term) also play a major role. 


\section{Use of the certified reference material and the 'Good Practice Guide'}

The certified reference material (CRM) can be used for two main purposes: (1) for verification of (calibrated) scratch-test instruments, giving a good indication of overall instrument performance and (2) for establishing control charts for monitoring instrument performance (including stylus) over an extended period. Clear and efficient instructions to potential users of the CRM are found in the 'Good Practice Guide' [7].

\subsection{Verification}

The certified ranges, as calculated in Eq. (1), give to a first approximation, the ranges within which $95 \%$ of critical loads should fall when testing a specimen on an average machine with an average stylus of those used in the certification campaign. However, it is apparent that certain specific combinations of machine, stylus and sample may produce sets of critical loads that lie outside the certified range. A user will want to verify one particular type of (calibrated) test machine fitted with any stylus conforming to ISO 6508-2, possibly at the extreme edge of the allowable range of radii. To achieve this, the user is required to scratch one of the reference samples, using the machine being examined, calculate (for each failure event) a critical load value and compare it with a range of critical loads or 'verification range' specified for the CRM, which includes an allowance for the effects of machine and stylus. Clearly, for the specified verification range to be tolerant to the use of different types of machines with different styli, it is larger than the certified range, $U_{\mathrm{c}}$ above. This is achieved by the inclusion of the instrument and stylus offsets as estimated by $u_{1}$ and $u_{\mathrm{ss}}$. The use of specific components of the verification range is described more fully in the Good Practice Guide.

In general the user is required to make 5 scratches according to the instructions for use. The user should ensure that the repeatability of his/her results (the standard deviation of the five values for each critical load) are similar to the $u_{\mathrm{ws}}$ values measured during the CRM-692 certification (Table 1) representative of an instrument of good performance. If statistical comparison of each of the critical loads measured $\left(\mathrm{Lc}_{1}, \mathrm{Lc}_{2}\right.$ and $\mathrm{Lc}_{3}$ ), results in the mean of each set of five and the standard deviations fall within the ranges given in the Good Practice Guide, then this is a good indication that the instrument is functioning well. If this is not the case, then the user should more carefully check the instrument components, particularly the stylus, and the calibration. The verification is, however, a statistical process at a confidence level less than $100 \%$. It is possible that the instrument and stylus may have significant, but counteracting defects, that keep the measured critical load values within the certified ranges. It is also possible that a combination of extreme values of (individually acceptable) offsets combine to push the results systematically outside the verification range. The use of the reference material for verification as described below is therefore not a $100 \%$ guarantee that the scratch-test instrument is operating in an optimum way, (but is roughly a $95 \%$ guarantee at the $95 \%$ confidence level). For these reasons, the reference material cannot be used to calibrate the scratch tester.

\subsection{Monitoring}

The reference sample can provide a sensitive monitor of instrument performance over time (control chart). To do this, the user should carry out the steps given in the instructions for use and monitor the evolution of mean critical loads measured and the standard deviations. This procedure only has value provided the same reference sample and stylus are used for the duration of the monitoring. The frequency of the monitor measurements depends on the other uses of the instrument being tested, bearing in mind that the expected stylus lifetime on the BCR-692 DLC coatings is approximately 400 scratches, and is likely to be less on higher friction coatings and with higher loads.

\section{Conclusions}

A CRM BCR-692 is available for verification and monitoring purposes. The reference samples are $30 \times 30 \times 5 \mathrm{~mm}^{3}$ steel coupons coated with a DLC coating, distributed in a reusable plastic box containing desiccant. The CRM presents three repeatable failure events at certified critical load intervals and can provide a good indication of overall performance, including stylus condition and calibration. It is also useful as a diagnostic tool, providing a means of sensitive monitoring of machine and stylus performance over extended periods. Considerable improvement of the quality of the used scratch styli was achieved by the strict control of all manufacturing steps.

\section{References}

[1] European Standard prEN1071-3:2000:E, Advanced technical ceramics-methods of test for ceramic coatings-Part 3: determination of adhesion and other mechanical failure modes by a scratch test, CEN Management Centre, Stassartstraat 36, B1050 Brussels, Belgium.

[2] J. Meneve, et al., Scratch adhesion testing of coated surfaceschallenges and new directions, K.L. Mittal (Ed.) VSP International Science Publishers, Zeist, The Netherlands, Adhesion Measurement of Films and Coatings, vol. 2, 2001, pp. 79106.

[3] European Commission-Standards, Measurements and Testing Program, Project 'A Certified Reference Material for the Scratch Test-REMAST', contract SMT4-CT98/2238, completed 31/12/2001. 
[4] Standard EN ISO 6508-2:1999, Metallic materials-Rockwell hardness test-Part 2: verification and calibration of testing instruments (scales A, B, C, D, E, F, G, H, K, N, T).

[5] European Commission-Standards, Measurements and Testing Program, Project 'Multimode Scratch Testing (MMST): Extension of Operation Modes and Update of Instrumentation', contract SMT4-CT97/2150, completed 2001.
[6] ISO document REMCO/WG 1 N 41 (Draft Guide 35, Certification of Reference Materials-General and statistical principles), ISO, Geneva, Switzerland, February 2002.

[7] N.M. Jennett, S. Owen-Jones, NPL Measurement Good Practice Guide No. 54 'The Scratch Test: Calibration, Validation and the use of a Certified Reference Material', NPL Materials Centre. 